\title{
Evaluation of a Low-Cost Strategy for Enumerating CD4 Lymphocyte Absolute Count and Percentage Using the FACSCalibur Flow Cytometer in HIV-Infected Patients from a Resource-Limited Setting
}

\author{
Gerardo Alvarez-Uria, ${ }^{1}$ Raghuprakash Reddy, ${ }^{2}$ Srinivasulu Reddy, ${ }^{2}$ \\ Praveen K. Naik, ${ }^{1}$ and Manoranjan Midde ${ }^{1}$ \\ ${ }^{1}$ Department of Infectious Diseases, Rural Development Trust Hospital, Kadiri Road, Bathalapalli 515661, India \\ ${ }^{2}$ Department of Microbiology, Rural Development Trust Hospital, Kadiri Road, Bathalapalli 515661, India
}

Correspondence should be addressed to Gerardo Alvarez-Uria, gerardouria@gmail.com

Received 12 September 2012; Accepted 27 September 2012

Academic Editors: L. Belec and J. Poudrier

Copyright () 2012 Gerardo Alvarez-Uria et al. This is an open access article distributed under the Creative Commons Attribution License, which permits unrestricted use, distribution, and reproduction in any medium, provided the original work is properly cited.

Enumeration of CD4 lymphocytes is essential for the clinical management of HIV-infected patients, but it can be difficult to afford in developing countries. In this study we evaluated a reagent reduction strategy for reducing the cost of enumerating CD4 cell absolute count and percentage using the FACSCalibur flow cytometer (Becton Dickinson). We compared the protocol recommended by the manufacturer with a protocol that used half of the usual amount of CD3/CD4/CD45 monoclonal antibody reagent in 100 samples from HIV-infected patients in a rural hospital in India. The concordance correlation coefficient between the two protocols was 0.976 for CD4 cell count and 0.984 for CD4 cell percentage. We did not find significant bias when performing Deming regression or Bland-Altman analysis. Sensitivity and specificity were $97 \%$ and $98.5 \%$ for identifying patients with less than $200 \mathrm{CD} 4$ cells $/ \mu \mathrm{L}, 98.1 \%$ and $93.8 \%$ for identifying patients with less than $350 \mathrm{CD} 4$ cells/ $\mu \mathrm{L}$, and $100 \%$ and $94.7 \%$ for identifying patients with less than $25 \%$ CD 4 cells, respectively. This reagent reduction strategy can be used for reducing the cost of enumerating CD4 lymphocytes in high-volume laboratories from resource-limited settings.

\section{Introduction}

In 2010, it was estimated that 34 million people were living with HIV/AIDS and more than 90\% were living in low and middle-income countries [1]. Enumeration of CD4 lymphocytes is essential for the clinical management of HIV-infected people. CD4 lymphocyte count can be used for initiating or stopping prophylaxis against opportunistic infections and for deciding when to initiate antiretroviral therapy against HIV [2].

Immunophenotyping by flow cytometer is the most accepted technology for enumeration of CD4 lymphocytes [3]. The FACSCalibur system (Becton Dickinson Biosciences, CA, USA) is a bench-top flow cytometer widely used in laboratories from developed countries and it is considered as the "gold standard" of CD4 counting [4]. However, the high cost of the reagents can be an important limitation for its use in resource-limited settings. In flow cytometry, monoclonal antibodies are the most expensive part of the reagents used to enumerate the absolute count and percentage of CD4 lymphocytes. The objective of this study is to evaluate a reagent reduction strategy for enumerating CD4 lymphocytes with the FACSCalibur system that used half of the usual amount of monoclonal antibodies.

\section{Methods}

The study was performed in the RDT Bathalapalli Hospital, Andhra Pradesh, India. After giving informed consent, peripheral blood was taken from $100 \mathrm{HIV}$-infected patients attending the Department of Infectious Diseases for 
TABLE 1: Concordance correlation coefficients, bias estimation by Deming regression and Bland-Altman analysis between protocol A and B.

\begin{tabular}{|c|c|c|c|c|}
\hline & $\begin{array}{c}\text { CCC } \\
(95 \% \mathrm{CI}) \\
\end{array}$ & $\begin{array}{c}y \text {-intercept } \\
(95 \% \mathrm{CI})\end{array}$ & $\begin{array}{c}\text { Slope } \\
(95 \% \text { CI })\end{array}$ & $\begin{array}{c}\text { Mean difference } \\
(95 \% \text { LOA })\end{array}$ \\
\hline CD4 cell count & $\begin{array}{c}0.976 \\
(0.966 \text { to } 0.985)\end{array}$ & $\begin{array}{c}8.68 \\
(-5.18 \text { to } 22.54)\end{array}$ & $\begin{array}{c}1.02 \\
(0.97 \text { to } 1.08)\end{array}$ & $\begin{array}{c}17.18 \\
(-85.88 \text { to } 120.24)\end{array}$ \\
\hline CD4 cell percentage & $\begin{array}{c}0.984 \\
(0.978 \text { to } 0.99)\end{array}$ & $\begin{array}{c}0.03 \\
(-0.53 \text { to } 0.59)\end{array}$ & $\begin{array}{c}1 \\
(0.97 \text { to } 1.03)\end{array}$ & $\begin{array}{c}0.01 \\
(-2.91 \text { to } 2.93)\end{array}$ \\
\hline
\end{tabular}

CCC: concordance correlation coefficient; CI: confidence interval; LOA: limits of agreement.

TABle 2: Sensitivity and specificity with Wilson's 95\% confidence intervals of protocol B to identify patients having $<200$ cells $/ \mu \mathrm{L}$, $<350$ cells $/ \mu \mathrm{L}$, and $<25 \%$ CD4 cells with protocol A.

\begin{tabular}{lcc}
\hline & Sensitivity & Specificity \\
& $\%(95 \% \mathrm{CI})$ & $\%(95 \% \mathrm{CI})$ \\
\hline$<200$ cells $/ \mu \mathrm{L}$ & 97 & 98.5 \\
& $(84.7-99.5)$ & $(92-99.7)$ \\
$<350$ cells $/ \mu \mathrm{L}$ & 98.1 & 93.8 \\
& $(89.9-99.7)$ & $(83.2-97.9)$ \\
$<25 \%$ CD4 cells & 100 & 94.7 \\
& $(95.5-100)$ & $(75.4-99.1)$ \\
\hline
\end{tabular}

CI: confidence interval.

enumerating the CD4 lymphocytes as per routine clinical management.

We compared the protocol recommended by the manufacturer (protocol A) against a protocol that required lesser quantity of reagent (protocol B). Protocol B is an improved version of a protocol suggested by the Application Team of $\mathrm{BD}$ in India. Both protocols $\mathrm{A}$ and $\mathrm{B}$ were performed according to manufacturer's standard operating procedures by trained technicians. Whole blood was collected in a single K3 EDTA tube for each patient and both protocols were performed the same day.

For protocol A, we introduced $20 \mu \mathrm{L}$ of CD3/CD4/CD45 monoclonal antibody reagent (BD TriTEST) and $50 \mu \mathrm{L}$ of whole blood in a tube with a lyophilized pellet having a calibrated quantity of fluorescent beads (BD TruCount). Tubes were incubated for 15 minutes at room temperature $\left(20-25^{\circ} \mathrm{C}\right)$ in a dark place before $450 \mu \mathrm{L}$ of lysing solution was added. The tube was incubated for 15 minutes at room temperature $\left(20-25^{\circ} \mathrm{C}\right)$ in a dark place again and then the sample was processed in the FACSCalibur system.

For protocol B, we introduced $10 \mu \mathrm{L}$ of CD3/CD4/CD45 monoclonal antibody reagent (BD TriTEST) and $25 \mu \mathrm{L}$ of whole blood in a plain polystyrene tube (BD Falcon). Tubes were incubated for 15 minutes at room temperature (20$25^{\circ} \mathrm{C}$ ) in a dark place before $450 \mu \mathrm{L}$ of lysing solution was added. The tube was incubated for 15 minutes at room temperature $\left(20-25^{\circ} \mathrm{C}\right)$ in a dark place again before $25 \mu \mathrm{L}$ of fluorescent bead solution (BD Liquid Counting Beads) was added. Then the sample was processed in the FACSCalibur system.

Statistical analysis was performed using Stata Statistical Software (Stata Corporation, Release 11, College Station, TX, USA). Bias was estimated using Deming regression [5]. Deming regression calculates the $95 \%$ confidence interval for the estimate of the intercept and the slope of a linear regression equation $(y=a[y-$ intercept $]+b$ [slope $x)$. If the value of the $y$-intercept is significantly different from 0 indicates a constant bias and if the value of the slope is significantly different from 1 indicates a proportional bias. The agreement between the two protocols was assessed by using the Altman-Bland method and concordance correlation coefficients (CCCs) [6].

\section{Results and Discussion}

The study included 100 samples from HIV-infected patients. The mean CD4 lymphocyte count with protocol A was 366 cells $/ \mu \mathrm{L}$ (range 21-1136, standard deviation 254) and the mean CD4 lymphocyte count with protocol B was 349 cells $/ \mu \mathrm{L}$ (range 23-1072, standard deviation 248). The mean CD4 lymphocyte percentage with protocol A was $18.4 \%$ (range 3-37, standard deviation 8.3) and the mean CD4 lymphocyte percentage with protocol B was $18.4 \%$ (range 3-39, standard deviation 8.3).

Estimation of bias and agreement between the two protocols are presented in Table 1. When performing Deming regression, we did not find any significant bias for CD4 cell count or CD4 cell percentage as the confidence intervals for $y$-intercepts included 0 and the confidence intervals for slopes included 1. The concordance correlation coefficient was slightly higher for CD4 cell percentage than for CD4 cell count. Correlation and Bland-Altman plots are shown in Figure 1. Bland-Altman analysis showed close agreement between the two protocols.

Sensitivities and specificities of protocol B for three clinically relevant cutoffs are presented in Table 2. Patients having less than $200 \mathrm{CD} 4$ cells/ $\mu \mathrm{L}$ need to initiate prophylaxis against Pneumocystis jirovecii pneumonia and this cutoff is useful for identifying patients in risk of opportunistic infections [2]. In HIV-infected patients, having less than $350 \mathrm{CD} 4$ cells $/ \mu \mathrm{L}$ is an indication for initiating antiretroviral therapy in patients older than 5 years and having less than 25\% CD4 lymphocytes is an indication for initiating antiretroviral therapy in children aged 2 to 5 years $[2,7]$.

Although the reagent reduction strategy has been successfully evaluated in previous studies [8], to our knowledge this is the first time that this strategy has been evaluated for the FACSCalibur system. The cost of FACSCalibur reagents is volume dependent but ranges from 3 to 7 United States dollars (USD) per test [4]. Using protocol B, the cost of the reagents can be reduced by a half, making the final cost per test lower than other low-cost technologies 


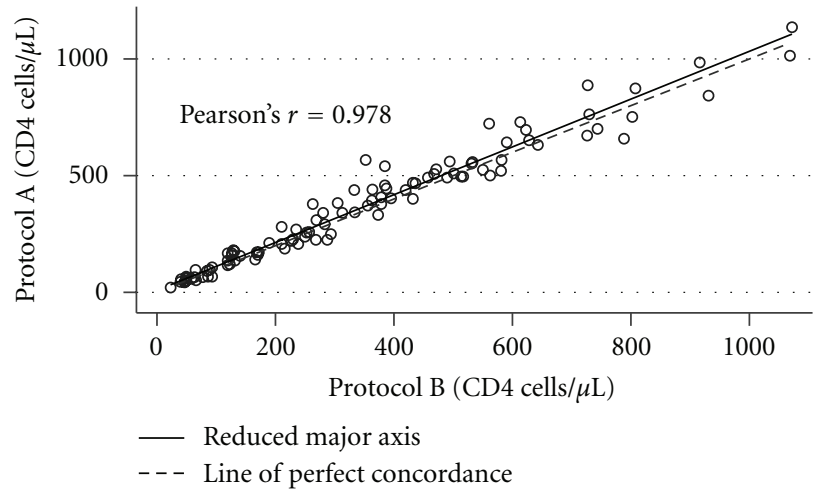

(a)

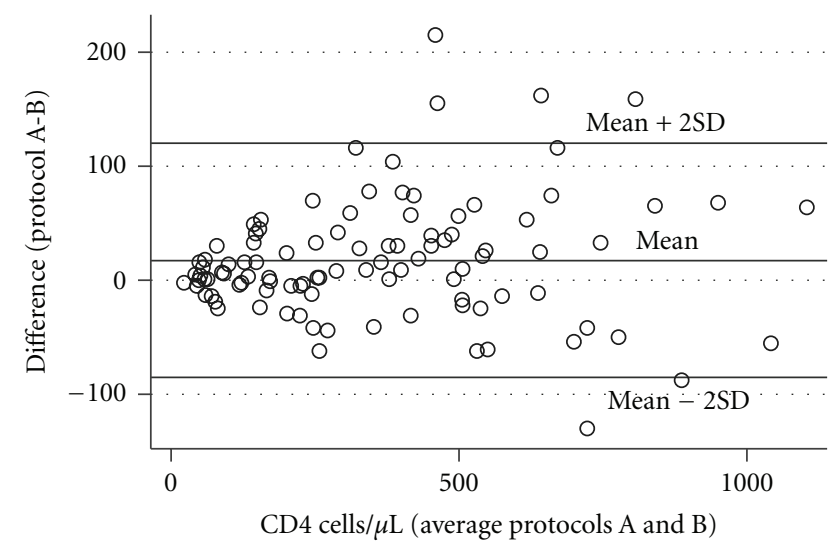

(c)

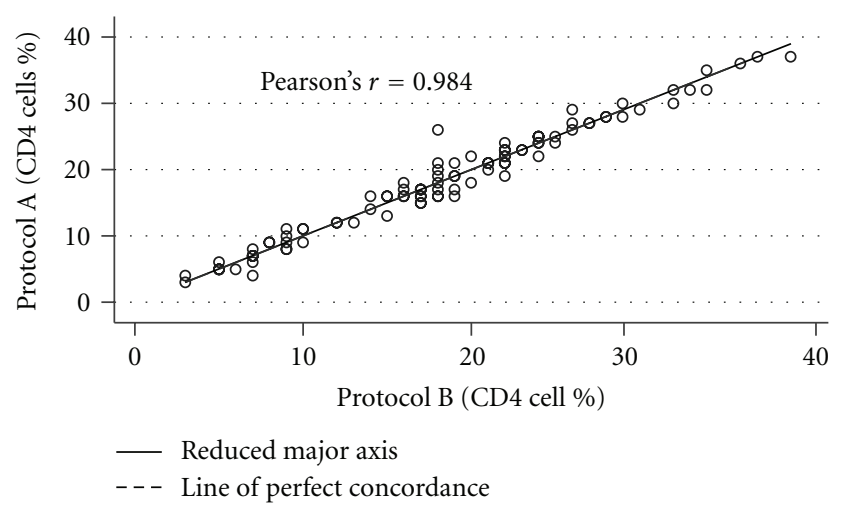

(b)

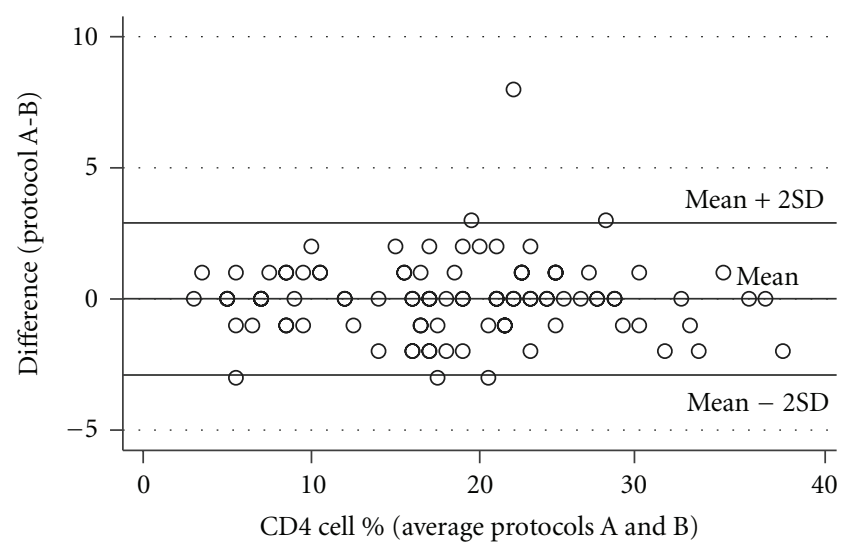

(d)

Figure 1: Correlation plots of CD4 cell count (a) and CD4 cell percentage (b), and Bland-Altman plots of CD4 cell count (c) and CD4 cell percentage (d) of protocol A versus protocol B.

[4]. The FACSCalibur system can be especially useful for laboratories receiving high volume of samples because it is a single platform system, does not require highly trained operators, and provides a convenient walk-away automation through a sample loader $[4,9]$. Although this cost reduction makes the FACSCalibur system a very interesting option for high volume laboratories, the cost of the instrument is about 75,000 USD [4], which is considerably higher than other technologies, so the system may not be feasible for laboratories receiving a low volume of samples in resourcelimited settings.

\section{Conclusions}

Protocol B is able to provide reliable results of CD4 cell count and percentage with half of usual amount of monoclonal antibody reagent. This reagent reduction strategy can be used for reducing the cost of enumerating CD4 lymphocytes with the FACSCalibur system.

\section{Conflict of Interests}

The authors declare no conflict of interests.

\section{Acknowledgments}

The authors would like to thank Susana Malumbres and Blanca Gomez-Escoda for their help in this paper.

\section{References}

[1] UNAIDS, Data tables, 2011.

[2] World Health Organization, Antiretroviral Therapy for HIV Infection in Adults and Adolescents, 2010.

[3] D. Barnett, B. Walker, A. Landay, and T. N. Denny, "CD4 immunophenotyping in HIV infection," Nature Reviews Microbiology, vol. 6, no. 11, pp. S7-S15, 2008.

[4] UNITAID, HIV/AIDS diagnostic technology landscape, 2012.

[5] K. Linnet, "Evaluation of regression procedures for methods comparison studies," Clinical Chemistry, vol. 39, no. 3, pp. 424432, 1993.

[6] H. X. Barnhart, M. J. Haber, and L. I. Lin, "An overview on assessing agreement with continuous measurements," Journal of Biopharmaceutical Statistics, vol. 17, no. 4, pp. 529-569, 2007.

[7] World Health Organization, Antiretroviral Therapy for HIV Infection in Infants and Children: Towards Universal Access, 2010.

[8] S. Buranapraditkun, S. Nookhai, S. Ubolyam, S. Sirivichayakul, P. Phanuphak, and K. Ruxrungtham, "Cost savings by reagent 
reduction in flow cytometry-based CD4+ T cell counts: an approach to improve accessibility for HIV management," Asian Pacific Journal of Allergy and Immunology, vol. 25, no. 1, pp. 8389, 2007.

[9] T. Peter, A. Badrichani, E. Wu et al., "Challenges in implementing CD4 testing in resource-limited settings," Cytometry B, vol. 74, supplement 1, pp. S123-S130, 2008. 


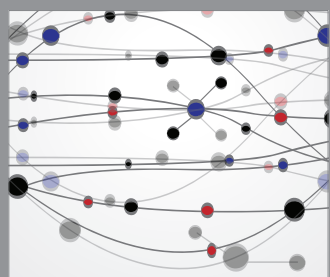

The Scientific World Journal
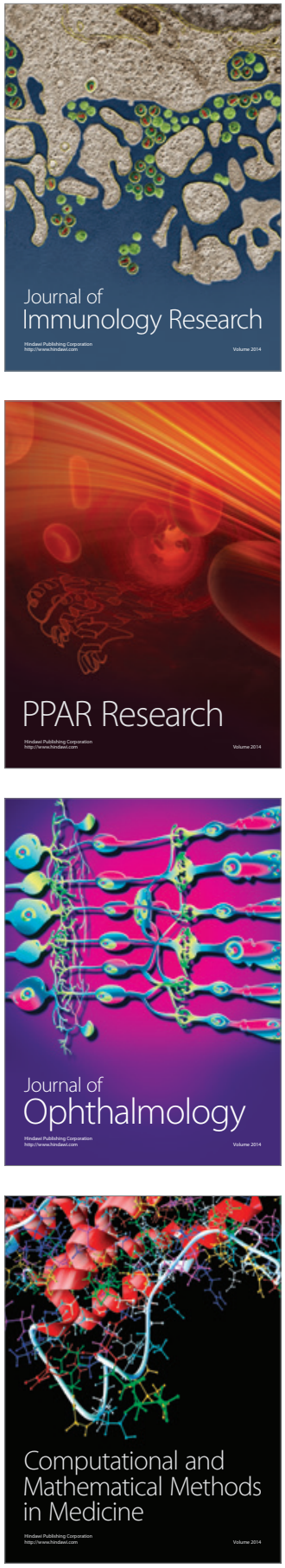

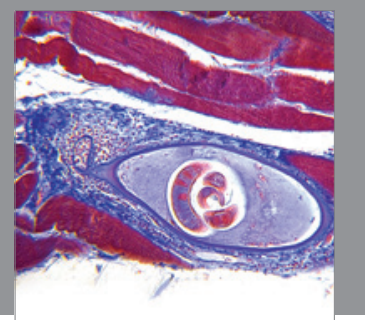

Gastroenterology

Research and Practice
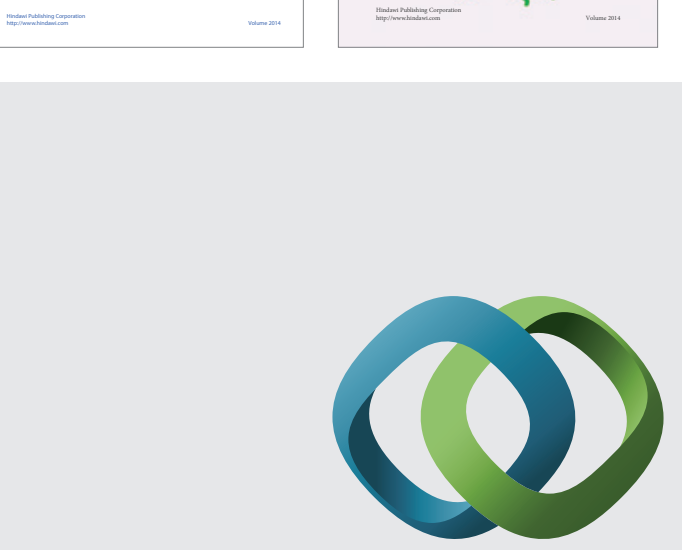

\section{Hindawi}

Submit your manuscripts at

http://www.hindawi.com
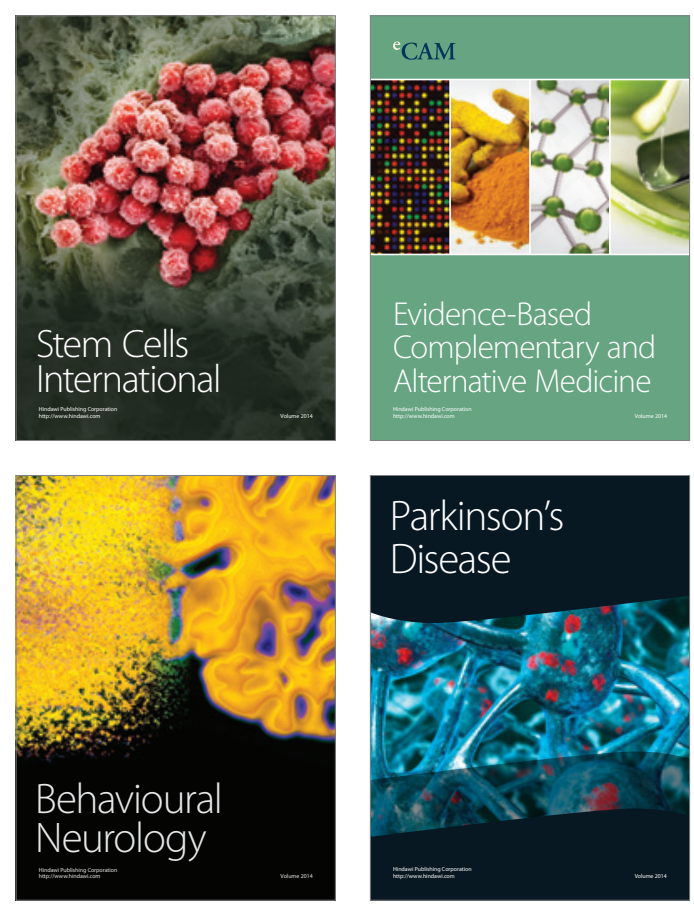

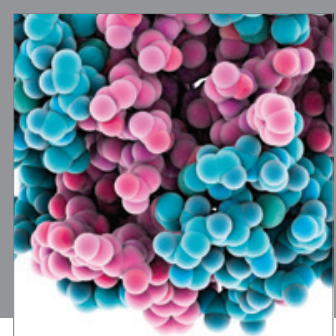

Journal of
Diabetes Research

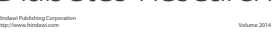

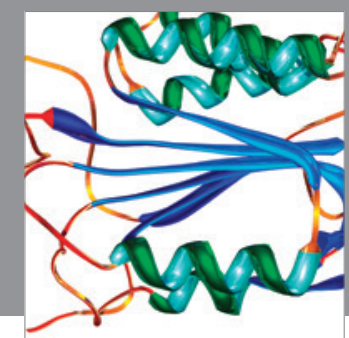

Disease Markers
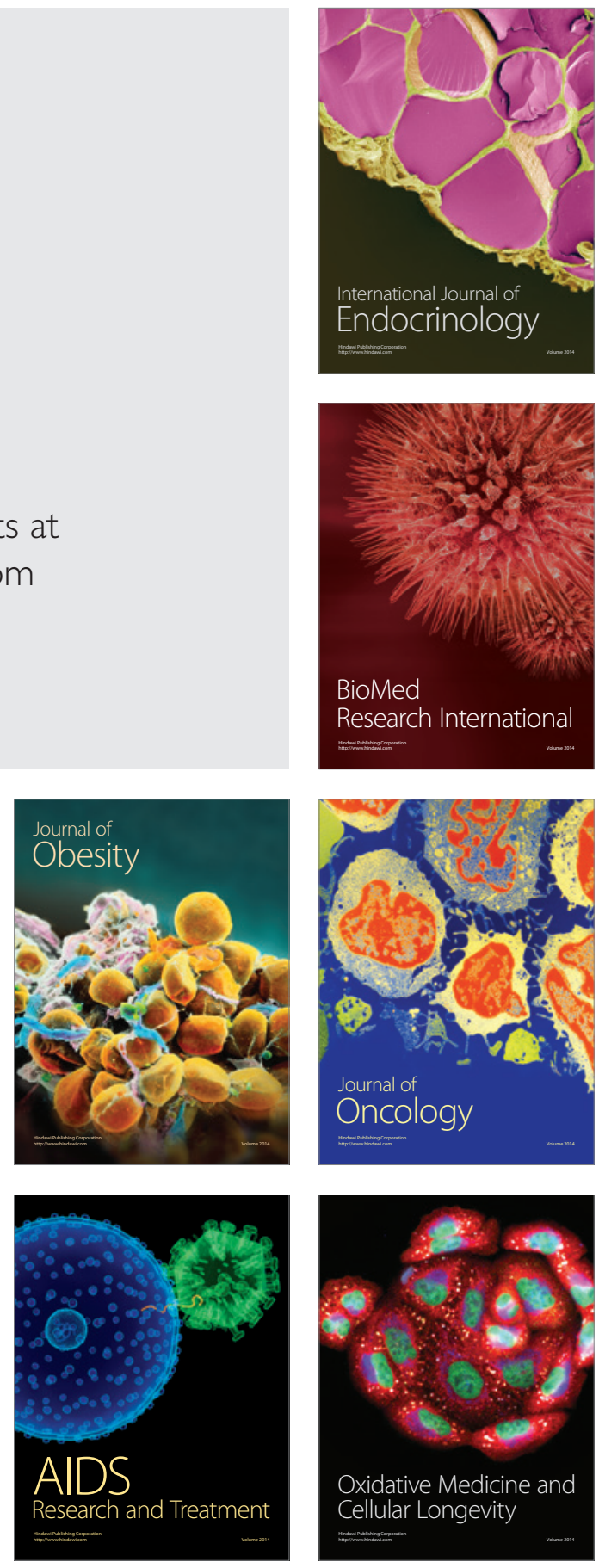